\title{
Caracterização de argilas de novos jazimentos situados em Parelhas/RN, Brasil, visando aplicação na indústria cerâmica
}

\section{Characterization of clays of deposits new located in Parelhas/RN, Brasil, aiming for application in the ceramic industry}

Sileide de Oliveira Ramos ${ }^{1}$, Gerbeson Carlos Batista Dantas ${ }^{1}$, Hélio de Lucena Lira ${ }^{2}$, Patrícia Mendonça Pimentel ${ }^{1}$, João Emanuel Araújo Marciano ${ }^{3}$

\footnotetext{
${ }^{1}$ Universidade Federal Rural do Semi-Árido, Campus Angicos 59515-000, Angicos, Rio Grande do Norte, Brasil. e-mail: sileide.ramos@ufersa.edu.br, gerbeson_dantas@hotmail.com, pimentelmp@ufersa.edu.br

${ }^{2}$ Universidade Federal de Campina Grande, 58109-970, Campina Grande, Paraíba, Brasil. e-mail: helio.lira@ufcg.edu.br

${ }^{3}$ Universidade Federal de Pernambuco, 50670-901, Recife, Pernambuco, Brasil.

e-mail: joao.emanuell@ufpe.br
}

\section{RESUMO}

O Estado do Rio Grande do Norte, mais especificamente no município de Parelhas, Brasil, a indústria cerâmica já é bastante consolidada, crescendo ano após ano. Entretanto, o estímulo do setor tem provocado acelerada exaustão das jazidas de argilas locais utilizadas pela indústria e, por conseguinte, ameaçando a manutenção da indústria cerâmica no município e na região Seridó. Por esta razão, tem-se aprofundado o interesse de buscar novos jazimentos de argilas, a fim de suprir as suas demandas atuais e futuras. Recentemente, foram descobertos novos jazimentos no município, todavia, estas argilas não têm sido devidamente caracterizadas, causando prejuízos tanto a qualidade final do produto, como ao seu pleno aproveitamento industrial. Assim, este trabalho tem como objetivo caracterizar física, química e mineralogicamente argilas extraídas de três novos jazimentos do município de Parelhas/RN, com a finalidade de classificá-las visando sua aplicação na indústria de cerâmica vermelha da região. As amostras foram beneficiadas por secagem a $60{ }^{\circ} \mathrm{C}$, seguida de moagem e peneiramento em malha de 200 mesh. Então, foram caracterizadas por fluorescência de raios-X, difração de raios-x, análise termogravimétrica e diferencial, análise granulométrica, perda ao fogo e plasticidade. Os constituintes das amostras identificados por fluorescência de raios $\mathrm{X}$ foram $\mathrm{SiO}_{2}, \mathrm{Al}_{2} \mathrm{O}_{3}, \mathrm{Fe}_{2} \mathrm{O}_{3}$, $\mathrm{MgO}, \mathrm{K}_{2} \mathrm{O}, \mathrm{CaO}$ e $\mathrm{TiO}_{2}$. Os difratogramas de raios-X revelaram a presença predominante dos argilominerais esmectita, feldspato, caulinita e quartzo nas amostras. A perda de massa total para as amostras variou entre $14 \%$ a $22,65 \%$. A distribuição de partículas variou entre 0,2 a $40 \mu \mathrm{m}$, com diâmetro médio entre 6 e $16 \mu \mathrm{m}$. As amostras são classificadas como altamente plásticas e apresentam viabilidade para serem utilizadas na indústria de cerâmica vermelha.

Palavras-chave: Argilas; Técnicas de caracterização; Parelhas/RN; Indústria Cerâmica.

\section{ABSTRACT}

The State of Rio Grande do Norte, more specifically in the municipality of Parelhas, Brazil, the ceramic industry is already well established, growing year after year. However, the industry stimulus has led to accelerated exhaustion of the local clay deposits used by the industry and, therefore, threatening the maintenance of the ceramics industry in the municipality and the Seridó region. For this reason, interest has been deepened in the search for new clay deposits in order to meet their current and future demands. Recently, new deposits have been discovered in the municipality, however, these clays have not been properly characterized, causing damage to both the final quality of the product and its full industrial use.Thus, this work aims to characterize physically, chemically and mineralogically clays extracted from deposits in the Parelhas/RN municipality, 
with the purpose of classifying them aiming to be applied in the ceramic industry of the region. The samples were beneficiated by drying at $60{ }^{\circ} \mathrm{C}$, followed by milling and sieving in 200 mesh sieves. Then, they were characterized by $\mathrm{x}$-ray fluorescence, $\mathrm{x}$-ray diffraction, thermogravimetric and differential analysis, particle size analysis, ignition loss and plasticity. The constituents of the samples identified by X-ray fluorescence were $\mathrm{SiO}_{2}, \mathrm{Al}_{2} \mathrm{O}_{3}, \mathrm{Fe}_{2} \mathrm{O}_{3}, \mathrm{MgO}, \mathrm{K}_{2} \mathrm{O}, \mathrm{CaO}$ and $\mathrm{TiO}_{2}$. The X-ray diffractograms revealed the predominant presence of the clay minerals smectite, feldspar, kaolinite and quartz in the samples. The total mass loss for the samples ranged from $14 \%$ to $22.65 \%$. The particle distribution ranged from 0.2 to $40 \mu \mathrm{m}$, with an average diameter between 3 and $7 \mu \mathrm{m}$. The samples are classified as highly plastic and are feasible for use in the red ceramic industry.

Keywords: Clays; Characterization techniques; Parelhas/RN; Ceramic industry.

\section{INTRODUÇÃO}

As argilas são materiais encontrados na natureza, bastante abundantes, de baixo custo de extração e processamento e altamente versáteis, além de ser, historicamente, o principal insumo utilizado na indústria de cerâmica vermelha [1-3]. Os materiais de cerâmica vermelha possuem um amplo campo de aplicação, sobretudo, na construção civil, como blocos de vedação e estruturais, telhas, tijolos, manilhas, agregado para concreto leve dentre outras inúmeras aplicações [4-6]. Somando as aplicações na construção civil, nas últimas décadas, as argilas têm sido utilizadas em aplicações mais avançadas, por exemplo, em suportes de catalisadores, como matéria-prima para cimentos, na indústria de refratários, adsorvente de contaminantes oleosos em processo de produção do biodiesel [7-8]. Em razão deste variado campo de aplicação, a indústria cerâmica tem crescido exponencialmente ano após ano [9].

As argilas são definidas como um material natural, de granulometria fina e que quando em contato com água, apresenta plasticidade. Em relação à composição, são materiais formados por argilominerais de silicatos e óxidos de alumínio, ferro e magnésio [4,10]. Em razão destas particularidades, as argilas são materiais muito heterogêneos cujas características dependem de sua formação geológica, bem como o local de extração [1,11], sendo, portanto, classificadas pela sua origem, composição química, limites de Atterberg e concentração dos argilominerais [4].

Não obstante, o Brasil é grande produtor de argilas, dispondo de milhares de jazimentos e empresas especializadas por todo território nacional, consumindo milhões de toneladas de argila todos os anos [5]. Entretanto, a maioria das argilas não é devidamente caracterizada, dificultando seu pleno aproveitamento industrial [12-13]. Nesse sentido, é indispensável realizar a identificação completa das características físicomineralógicas das argilas, uma vez que as propriedades do produto são dependentes das características das matérias-primas [5]. Somando-se a isso, reconhecer a caracterização como etapa do processo de obtenção dos produtos cerâmicos, além de adequar os produtos resultantes deste processo às exigências internacionais [1416], agrega valor à cerâmica, visto que há um aumento da qualidade e homogeneização das propriedades do produto final $[7,17]$.

No Estado do Rio Grande do Norte, mais especificamente no município de Parelhas, a indústria cerâmica já é bastante consolidada, exercendo grande importância socioeconômica, já que esta é responsável pela geração de centenas de empregos, como também é grande polo fornecedor de materiais estruturais para todo o país, fomentando a economia da região. Entretanto, o estímulo do setor tem provocado acelerada exaustão das jazidas locais utilizadas pelo setor e, por conseguinte, ameaçando a manutenção da indústria cerâmica no município e na região Seridó.

Por esta razão, tem-se aprofundado o interesse da indústria de cerâmica vermelha local no sentido de buscar novos jazimentos de argilas, a fim de suprir as suas demandas atuais e futuras. Recentemente, foram descobertos novos jazimentos no município, todavia, estas argilas não têm sido devidamente caracterizadas e portanto, não há registros significativos de caracterização dessas matérias-primas, causando prejuízos tanto a qualidade final do produto, como ao seu pleno aproveitamento industrial.

Nesse sentido, este trabalho tem como objetivo caracterizar física, química e mineralogicamente argilas extraídas de três novos jazimentos situados no município de Parelhas/RN, Brasil, com a finalidade de classificá-las visando sua aplicação na indústria de cerâmica vermelha da região.

\section{MATERIAIS E MÉTODOS}

\subsection{Materiais}

Para realização deste trabalho, foram utilizadas três amostras de argilas extraídas de três novos jazimentos do 
município de Parelhas, Estado do Rio Grande do Norte, Brasil, aqui identificadas como A, B e C.

\subsection{Métodos}

Inicialmente, as amostras coletadas foram beneficiadas por meio da realização do destorroamento, secagem em estufa a $60{ }^{\circ} \mathrm{C}$, moagem em moinho martelo e peneiramento manual em peneira de malha 30 mesh $(0,6$ $\mathrm{mm})$ e 80 mesh $(0,18 \mathrm{~mm})$, respectivamente. Em seguida, as amostras passaram por moagem em moinho galga, seguido de peneiramento em peneira vibratória em malha 200 mesh $(0,074 \mathrm{~mm})$ e, posteriormente, foram realizadas as caracterizações: química, mineralógica, térmica e granulométrica.

\subsection{Caracterização}

A caracterização das amostras das argilas foi efetuada por meio das seguintes técnicas: difração de raio-x, por meio do difratômetro de raios $\mathrm{X}$ da Shimadzu, modelo XRD-6000, com radiação $\mathrm{K} \alpha$ de $\mathrm{Cu}(40 \mathrm{kV} / 30 \mathrm{~mA})$, velocidade do goniômetro de $2{ }^{\circ} \mathrm{C} / \mathrm{min}$, passo de $0,02^{\circ}$ e varredura angular de $2 \theta$ entre 5 a $60^{\circ}$; análise termogravimétrica e diferencial, obtidas em uma termobalança, marca TA Instruments, modelo SDT 2960; espectroscopia de fluorescência de raios X, pelo espectrômetro de fluorescência de raios X por energia dispersiva (EDX), da marca Shimadzu, modelo EDX-700; análise granulométrica por intermédio de um granulômetro CILAS modelo $1064 \mathrm{LD}$; pelos limites de Attemberg [18-20] e perda ao fogo a $1000^{\circ} \mathrm{C}$.

\section{RESULTADOS E DISCUSSÃO}

A composição química das amostras de argilas A, B e C é apresentada na Tabela 1. A partir dos resultados observa-se que as argilas são compostas principalmente por óxido de silício $\left(\mathrm{SiO}_{2}\right)$, com teores variando entre 45,13 a 58,65\% sendo maior na argila C. Com relação ao teor de alumina $\left(\mathrm{Al}_{2} \mathrm{O}_{3}\right)$, que representou o segundo maior componente das argilas, identificou-se que os teores variaram entre $16,81 \%$ a $39,19 \%$, sendo maior na argila A. Estes resultados foram similares com outros trabalhos encontrados na literatura [3, 10, 21-23]. A relação $\mathrm{SiO}_{2} / \mathrm{Al}_{2} \mathrm{O}_{3}$ foi aproximadamente 3:1 para as amostras, exceto para amostra A, que apresentou relação aproximada de 1:1. A relação $\mathrm{SiO}_{2} / \mathrm{Al}_{2} \mathrm{O}_{3}$ é frequentemente associada ao empilhamento das camadas octaédricas e tetraédricas, das distâncias interplanares das camadas e das transições isomórficas [10]. Essas informações estão associadas à maioria das estruturas fundamentais dos argilominerais conhecidos e, portanto, podem ser utilizadas na classificação destes. A relação de 1:1 apresentada pela argila A é característico das argilas do grupo da caulinita, composta por uma camada tetraédrica dos silicatos e uma camada octaédrica dos aluminatos (na forma de hidróxidos) [10]. Enquanto a relação 3:1, é característica das argilas do grupo da esmectita, composta por duas camadas tetraédricas dos silicados e uma octaédrica dos aluminatos, unidas por oxigênios [10]. Em relação ao teor de $\mathrm{Fe}_{2} \mathrm{O}_{3}$ apresentado nas amostras $\mathrm{B}$ e C, estes variaram entre 8 e $12 \%$, podendo estar relacionado com as substituições isomórficas na camada octaédrica da esmectita, assim como, pelos minerais acessórios [24]. Os demais óxidos componentes das amostras de argila apresentam-se com teores inferiores a 5\%, comportando-se como fundentes quando submetidos a tratamento térmico [25]. Com relação à perda ao fogo a $1000^{\circ} \mathrm{C}$, as amostras apresentaram perdas de massa de $13,27 \%, 8,63 \%$ e 8,26\% para as argilas A, B e C, respectivamente. As perdas observadas estão relacionadas com a evaporação de água, seja dos vazios, adsorvida ou combinada, queima da matéria orgânica e a perda das hidroxilas dos argilominerais componentes das amostras [4].

Tabela 1: Composição química das amostras de argila

\begin{tabular}{|c|c|c|c|c|c|c|c|c|c|c|}
\hline AMOSTRAS & $\mathbf{P F}$ & $\mathrm{SiO}_{2}$ & $\mathrm{Al}_{2} \mathbf{O}_{3}$ & $\mathbf{M g O}$ & $\mathbf{K}_{2} \mathbf{O}$ & $\mathbf{F e}_{2} \mathbf{O}_{3}$ & $\mathbf{C a O}$ & $\mathbf{N a}_{2} \mathbf{O}$ & $\mathbf{T i O}_{2}$ & $\begin{array}{c}\text { OUTROS } \\
\text { ÓXIDOS }\end{array}$ \\
\hline $\mathrm{A}$ & 13,27 & 45,13 & 39,19 & 0,94 & 0,87 & 0,46 & - & - & - & 0,14 \\
\hline $\mathrm{B}$ & 8,63 & 53,65 & 16,81 & 2,76 & 0,53 & 11,37 & 4,75 & - & 1,07 & 0,43 \\
\hline $\mathrm{C}$ & 8,26 & 58,65 & 17,20 & 2,54 & 0,49 & 8,98 & 1,17 & 1,37 & 0,81 & 0,53 \\
\hline
\end{tabular}

Os difratogramas de raios-x das amostras de argilas estão ilustrados na Figura 1. Os difratogramas revelaram a presença das reflexões características dos argilominerais caulinita (JCPDS: 78-2110), quartzo (JCPDS: 46-1045), feldspato (JCPDS: 89-8575) e esmectita (JCPDS: 13-0135). A amostra A foi predominantemente caulinita, apresentando pico principal entre $10^{\circ}<2 \theta<15^{\circ}$. A presença em maior percentual da caulinita $\left(\mathrm{Al}_{2} \mathrm{Si}_{2} \mathrm{O}_{5}(\mathrm{OH})_{4}\right)$ na amostra $\mathrm{A}$, em comparação com as demais amostras, é corroborado com o re- 
sultado da análise química, uma vez que o elevado teor de $\mathrm{Al}_{2} \mathrm{O}_{3}$ e a relação $\mathrm{SiO}_{2} / \mathrm{Al}_{2} \mathrm{O}_{3}$ de aproximadamente 1:1 nesta amostra está associado a presença predominante do argilomineral caulinita [26]. Além disso, foram identificados quartzo e feldspato na amostra A, enquanto as outras amostras apresentaram além de quartzo, a presença predominante da esmectita (JCPDS: 13-0135) entre $2,5^{\circ}<2 \theta<7,5^{\circ}$, característico de argilas esmectíticas [24]. A presença principal da caulinita (amostra A) e esmectita (amostras B e C) é interessante, em razão de que estes argilominerais conferem a plasticidade necessária para que as amostras sejam conformadas com eficiência [27].

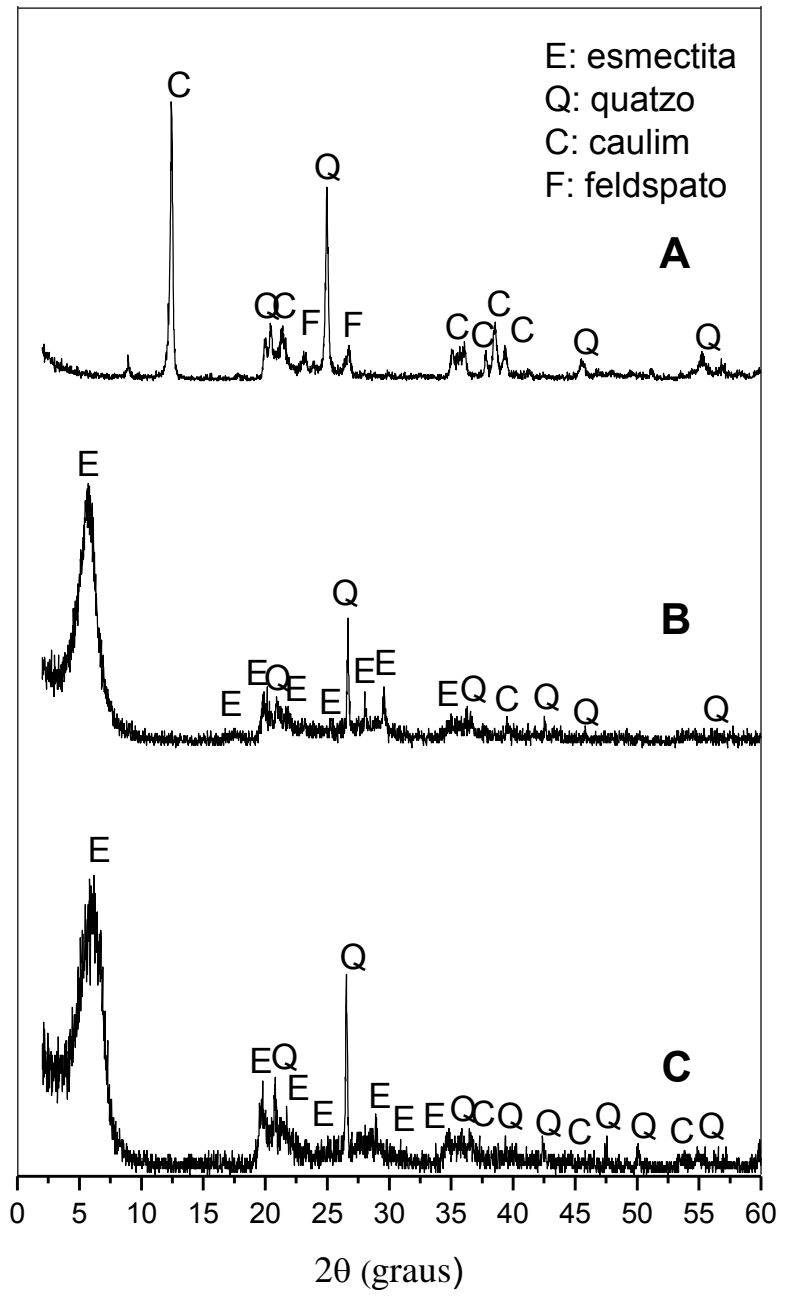

Figura 1: Difratogramas de raios $\mathrm{X}$ das amostras de argilas

A Figura 2 apresenta as curvas termogravimétricas das três amostras de argilas estudadas. Essas curvas evidenciam valores de perda de massa das amostras da ordem de 14, 22,65 e 22, 47\% para as amostras A, B e C, respectivamente. Para a amostra A, a perda de massa é menor que nas outras amostras e ocorre em temperaturas entre $400^{\circ} \mathrm{C}$ e $600^{\circ} \mathrm{C}$. Esse comportamento é esperado para argilas com elevado teor de caulinita. Nessas temperaturas inicia-se a desidroxilação da caulinita, que consiste na remoção da água combinada, seguida da transformação em metacaulinita [26, 28-29]. Este resultado está em conformidade com o pico endotérmico fornecido pela curva DTA da amostra A. Em acréscimo, observa-se um pico exotérmico em $997^{\circ} \mathrm{C}$ na curva DTA da amostra A referente a formação inicial da mulita [26]. Já para as amostras B e C, a perda de massa ocorre em três intervalos. O primeiro intervalo compreendido entre 0 e $200{ }^{\circ} \mathrm{C}$, é caracterizado pela evaporação de água, volatilização de gases adsorvidos e queima da matéria orgânica [29], cuja ocorrência pode ser ratificada pelo pico endotérmico inicial em $88^{\circ} \mathrm{C}$, observado nas curvas DTA das amostras B e C. A segunda faixa ocorre entre 200 a $680^{\circ} \mathrm{C}$. Nota-se que há outro pico endotérmico em $487^{\circ} \mathrm{C}$, que pode ser relativo a decomposição de argilominerais, especialmente, pela desidroxilação do aluminossilicato, bem como pela decomposição térmica do carbonato de cálcio em óxido de cálcio e gás $[10,25,30]$. Em $700^{\circ} \mathrm{C}$, a perda de massa alcança o patamar de estabilidade, correspondendo ao terceiro intervalo. 

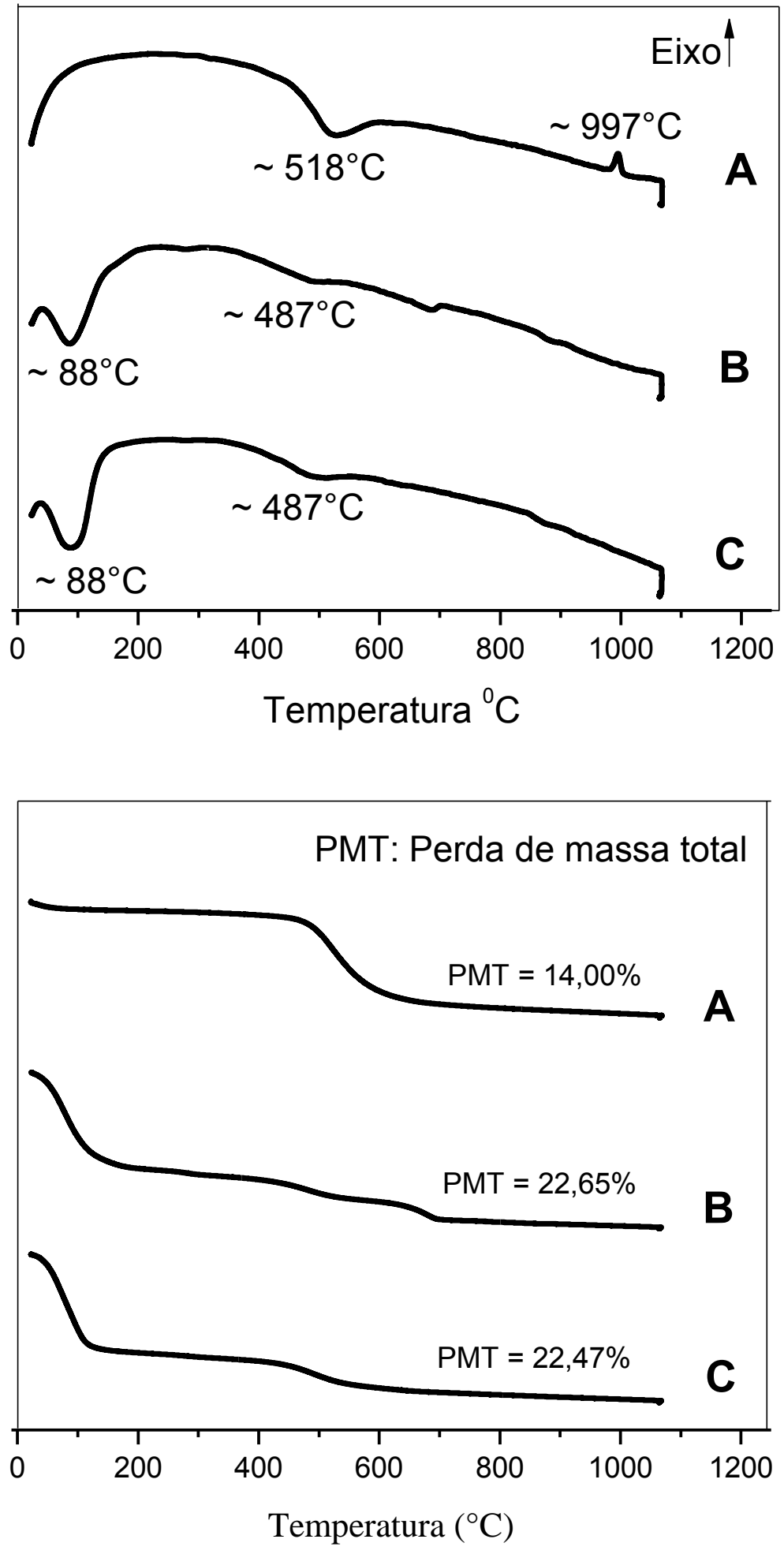

Figura 2: Curvas termogravimétricas e diferenciais das argilas

A Tabela 2 evidencia os valores de limite de plasticidade (LP), liquidez (LL) e índice de plasticidade (IP) das amostras analisadas. De acordo com os dados das propriedades físicas das argilas, observa-se que as argilas B e C apresentaram os maiores valores de limites de liquidez, plasticidade e de índice de plasticidade. Este comportamento está relacionado com a constituição destas argilas, uma vez que são predominantemente esmectíticas. A classificação das argilas pelo método de Casagrande encontra-se determinadas pelos seguintes intervalos: quando as argilas apresentam $1<\mathrm{IP} \leq 7$ são consideradas fracamente plásticas; quando as argi- 
las apresentam $7<$ IP $\leq 15$ são consideradas medianamente plásticas e as argilas com IP $>15$ são fortemente plásticas [13, 31]. Assim sendo, as argilas, objetos deste trabalho, são classificadas como altamente plástica, o que é um bom resultado para indústria cerâmica que recomenda índices de plasticidade no valor mínimo de 15. Portanto, as argilas investigadas nesse trabalho apresentam viabilidade para ser aplicadas na indústria cerâmica [32]. Outros trabalhos encontraram resultados similares de índice de plasticidade e justificam o uso das argilas na indústria cerâmica como matérias-primas, devido estas apresentarem plasticidade suficiente para serem conformadas, sem que haja a necessidade de aditivos plastificantes. O resultado é traduzido em economia [33-34], somando-se ao fato da elevada plasticidade estar associada a produtos com uma maior resistência mecânica após prensagem [35].

Tabela 2: Limites de Atterberg das amostras de argila

\begin{tabular}{|c|c|c|c|}
\hline AMOSTRAS & LL & LP & IP \\
\hline A & 45,72 & 28,17 & 17,55 \\
\hline B & 60,21 & 44,94 & 15,27 \\
\hline C & 84,12 & 54,67 & 29,45 \\
\hline
\end{tabular}

Os resultados da distribuição granulométrica e diâmetros característicos podem ser vistos na Figura 3 e na Tabela 3, respectivamente. Observa-se que as amostras apresentaram dois perfis de distribuição granulométrica, sendo bimodais para as amostras A e C e a amostra B, monomodal. De maneira geral, as argilas apresentaram distribuição de partículas entre $2 \mu \mathrm{m}$ a $100 \mu \mathrm{m}$, concentrando-se entre $2 \mu \mathrm{m}$ e $40 \mu \mathrm{m}$. Quanto aos diâmetros característicos, a Tabela 3 denota que estes são menores que $20 \mu \mathrm{m}$ para todas as amostras. A partir disto, é possível fazer conexão com os resultados de plasticidade, uma vez que a plasticidade das argilas está diretamente correlacionada com a granulometria, ou seja, quanto menor for a granulometria das partículas maior será a plasticidade, pois a área específica aumenta com a diminuição da granulometria [10]. Somandose a isso, argilas constituídas por elevados teores de argilominerais esmectíticos apresentam elevada finura e presença de matéria orgânica na forma de coloides protetores, que terminam por favorecer o desenvolvimento de plasticidade [5]. Os comportamentos supracitados explicam a razão das argilas apresentarem desempenho altamente plástico, especialmente a argila $\mathrm{C}$, que além de ser predominantemente esmectítico, apresentou maior plasticidade, menor diâmetro médio e concentração de partículas inferior as demais (inferior a $20 \mu \mathrm{m}$ ).

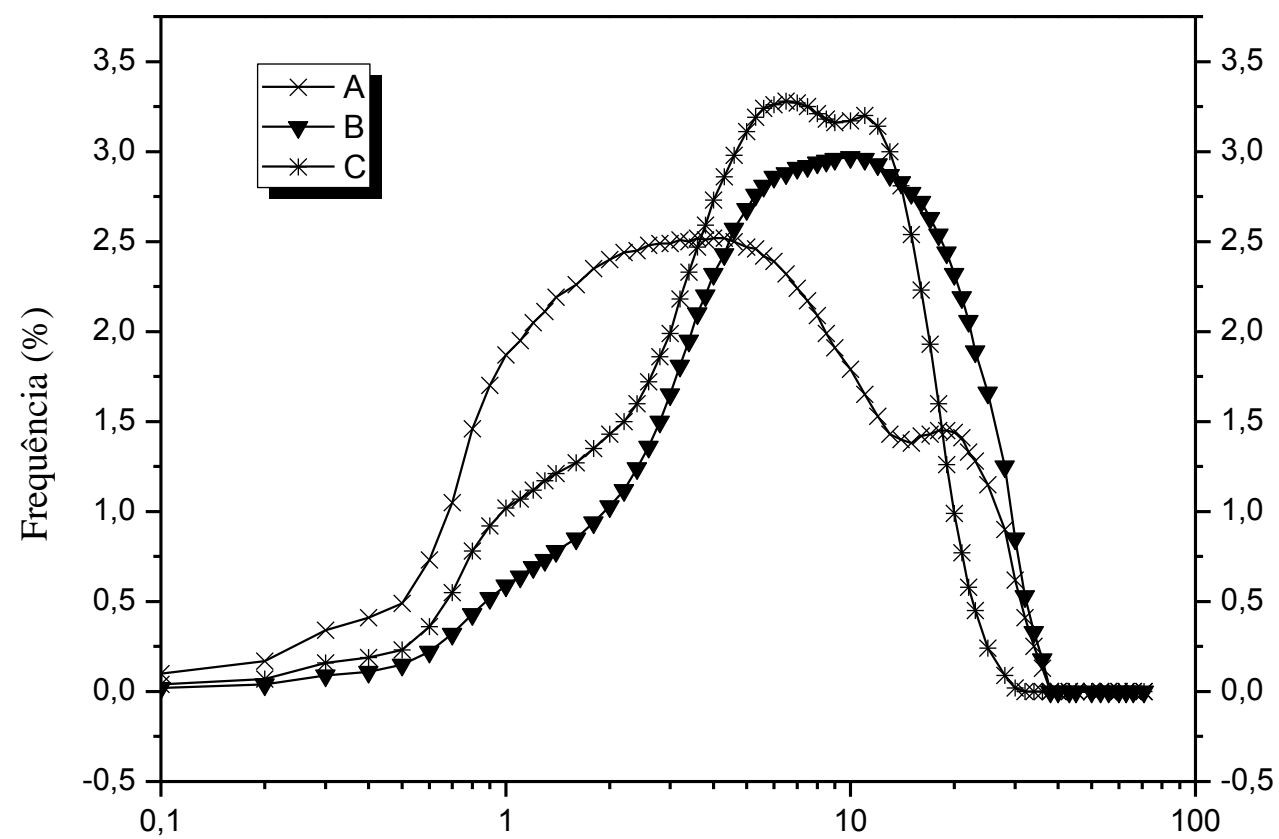

Diâmetro de Partícula $(\mu \mathrm{m})$

Figura 3: Distribuição granulométrica das argilas. 
Tabela 3: Diâmetros característicos das argilas.

\begin{tabular}{|c|c|c|c|}
\hline DIÂMETROS & A & B & C \\
\hline D10 $(\mu \mathrm{m})$ & 0,66 & 1,53 & 1,03 \\
\hline D50 $(\mu \mathrm{m})$ & 3,16 & 6,97 & 5,29 \\
\hline D90 $(\mu \mathrm{m})$ & 15,69 & 19,65 & 13,97 \\
\hline Dmédio $(\mu \mathrm{m})$ & 12,40 & 15,76 & 6,55 \\
\hline
\end{tabular}

A partir dos resultados obtidos, a amostra A pode ser classificada como uma argila do tipo caulinita em razão da sua composição apresentar elevado teor de $\mathrm{Al}_{2} \mathrm{O}_{3}(39,19 \%)$, resultando em uma relação $\mathrm{SiO}_{2} / \mathrm{Al}_{2} \mathrm{O}_{3}$ de aproximadamente 1:1, característico das argilas do grupo da caulinita, devido ao empilhamento das camadas tetraédricas dos silicatos e octaédrica dos aluminatos [10]. Somando-se a isso, a amostra A apresenta reflexão principal característica da caulinita entre $10^{\circ}<2 \theta<15^{\circ}$, sendo esta estreita e intensa, ou seja, com elevada cristalinidade, perda de massa superior a $400{ }^{\circ} \mathrm{C}$, o que é característico deste tipo de argila [26, 28-29, 36]. Já a amostra B foi classificada como argila do tipo esmectita pelos elevados teores de $\mathrm{SiO}_{2}$, relação $\mathrm{SiO}_{2} / \mathrm{Al}_{2} \mathrm{O}_{3}$ de aproximadamente 3:1, o que é característico deste tipo de argila, conforme discutido anteriormente [10,23]. A reflexão principal característica da amostra B está entre $2,5^{\circ}<2 \theta<7,5^{\circ}$ [24], perda de massa inicial acentuada e elevados teores de limites de liquidez [33-34], semelhante à amostra C, que também foi classificada como esmectita, porém, com maior valor de plasticidade.

\section{CONCLUSÕES}

A partir deste estudo, evidencia-se que as amostras de argilas de jazimentos de Parelhas/RN são compostas pelos argilominerais caulinita, quartzo, feldspato e esmectita. Apresentam distribuição granulométrica bimodal (argila A e C) e monomodal (argila B). Quanto aos limites de Atterberg, as amostras foram classificadas como altamente plásticas. De acordo com os resultados, a argila A pode ser classificada como caulinita, as argilas B e C como esmectitas. Por fim, as amostras apresentam potencial para serem utilizadas como matérias-primas na indústria de cerâmica vermelha, em razão da sua adequada composição mineralógica, distribuição granulométrica conveniente e desempenho altamente plástico, favorecendo a conformação dos materiais, a queima e o desempenho final do material.

\section{AGRADECIMENTOS}

Os autores agradecem ao Laboratório de Tecnologia de Materiais - LTM, Laboratório de Caracterização de Materiais - LCM, Laboratório de Reciclagem, Laboratório Multidisciplinar de Materiais e Estruturas Ativas - LAMMEA da Universidade Federal de Campina Grande pela cessão do espaço e realização das análises contidas neste trabalho.

\section{BIBLIOGRAFIA}

[1] REDDY, D., LEE, S.M., KIM, J.O., “A Review on emerging application of natural sericite and its composites”,World Applied Sciences Journal, v. 27, n.11, pp.1514, 2013.

[2] ROCHA, F., SUAREZ, P., GUIMARÃES, E., “Argilas e suas Aplicações em Utensílios e Materiais Cerâmicos”, Revista Virtual Química, v.6, n.4, pp.1105, 2014.

[3] ACEVEDO, N.I.A., ROCHA, M.C.G., BERTOLINO, L.C., "Mineralogical characterization of natural clays from Brazilian Southeast region for industrial applications", Cerâmica, v.63, n.366, pp.253-262, 2017.

[4] BRITO, P., ALMEIDA, E. P., NEVES, G. A., et al., “Avaliação de novos depósitos de argilas do Estado da Paraíba visando sua aplicação como matérias-primas cerâmicas”, Cerâmica, v.61, n.360, pp.391-398, 2015.

[5] RAMOS, S. O., MACEDO, R. S., CARTAXO, J. M., et al., "Caracterização de argilas usadas para cerâmica estrutural”,Revista Eletrônica de Materiais e Processos, v.5, n.3, pp.65-70, 2010.

[6] SILVA, A.L., LUNA, C.B.B., CHAVES, A.C.C., et al., "Caracterização tecnológica de novos depósitos de argilas da região sul do Amapá visando aplicações na indústria Cerâmica", Revista Matéria, v.22, n.1, pp.1-12, 2017.

[7] GONÇALVES, W.P., SILVA, V.J., GOMES, J., et al., “Avaliação da influência de diferentes tratamentos térmicos sobre as transformações de fases esmectitas", Cerâmica, v.60, n.355, pp.316-322, 2014.

[8] SILVA, R.A.O., SILVA, M.C.C., SOUSA, H.R., et al., "Prospecção tecnológica do uso de argila vermiculita como adsorvente de contaminantes oleosos em processo de produção do biodiesel", Cadernos de Pros- 
pecção, v.8, n.3, pp.544-551, 2015.

[9] WILLIAMS, L.B., HAYDEL, S.E., "Evaluation of the medicinal use of clay minerals as antibacterial agentes", International Geology Review, v.52, n.7/8, pp.745-770, 2010.

[10] SANTOS, P.S., Ciência e tecnologia de argilas, v.1, 2ed, Edgar Blücher, S. Paulo, SP 1992. 397p.

[11] PEREIRA, I.D.S., LISBOA, V.N.F., SILVA, I.A., et al., "BentoniteClaysfrom Sossego, Paraiba, Brazil: PhysicalandMineralogicalCharacterization", Materials Science Forum, v.798-799,pp.50-54, 2014.

[12] FORMIGA, F.L., ANDRADE, J.C.S., ARAÚJO, P.A.S., et al., "Indústrias cerâmicas da região do Seridó (RN): Panorama sócio-econômico e caracterização tecnológica de matérias-primas", Cerâmica Industrial, v.18, n.4, pp.30-36, 2013.

[13] MAESTRELLI, S.C., ROVERI, C.D., NUNES, A.G.P., et al., "Estudo de caracterização de argilas não plásticas da região de Poços de Caldas, MG", Cerâmica, v.59, n.350, pp.242-248, 2013.

[14] HAJJAJI, M., KACIM, S., BOULMANE, M., "Mineralogy and firing characteristics of a clay from the valley of Ourika (Morocco)", Applied Clay Science, v.21, n.3-4, pp.203- 212, 2002.

[15] CULTRONE, G., SIDRABA, I., SEBASTIÁN, E., "Mineralogical and physical characterization of the bricks used in the construction of the "Triangul Bastion", Riga (Latvia)", Applied Clay Science, v.28, n.1-4, pp.297-308, 2005.

[16] AJAM, L., OUEZDOU, M.B., FELFOUL, H.S., et al.,"Characterization of the Tunisian phosphogypsum and its valorization in clay bricks",Construction and Building Materials,v. 23, n.10, pp.3240-3247, 2009.

[17] DUTRA, R.P.S., VARELA, M.L.V., NASCIMENTO, R.M., et al., "Avaliação da Potencialidade de Argilas do Rio Grande do Norte - Brasil”, Cerâmica Industrial, v.11,n.2, pp.42-46, 2006.

[18] ASSOCIAÇÃO BRASILEIRA DE NORMAS TÉCNICAS. NBR 6459. Determinação do limite de liquidez - método de ensaio, Rio de Janeiro, RJ, Brasil, 1984.

[19] ASSOCIAÇÃO BRASILEIRA E NORMAS TÉCNICAS. NBR 6457 - Amostras de solos - preparação para ensaios de compactação e caracterização. Rio de Janeiro, RJ, Brasil, 2016.

[20] ASSOCIAÇÃO BRASILEIRA E NORMAS TÉCNICAS. NBR 7180. Determinação do limite de plasticidade - método de ensaio, Rio de Janeiro, RJ, Brasil, 1984.

[21] SILVA, I.A., COSTA, J.M.R., MENEZES, R.R., et al.,"Studies of new occurrences of bentonite clays in the State of Paraíba for use in water based drilling fluids", Revista Escola de Minas, v. 66, n.4, pp.485-491, 2013.

[22] OLIVEIRA, C. I. R., ROCHA, M. C. G., SILVA, A. L. N., et al., "Characterization of bentonite clays from Cubati, Paraíba (Northeast of Brazil)",Cerâmica, v. 62, n.363, pp. 272-277, 2016.

[23] OIKONOMOPOULOS, I.K., PERRAKI, M., TOUGIANNIDIS, N., et al., "Clays from Neogene Achlada lignite deposits in Florina basin (Western Macedonia, N. Greece): A prospective resource forthe ceramics industry", Applid Clay Science, v.103, pp.1-9, 2015.

[24] PEREIRA, I. D. S., SILVA, I. A., CARTAXO, J. M., et al., "Estudos de caracterização dos novos depósitos de argilas esmectíticas do município de Sossego, PB”, Cerâmica, v.60, n.354, pp.223-230, 2014.

[25] CARGNIN, M., SOUZA, S.M.A.G., SOUZA, A.A.U., et al., "Determinação de parâmetros cinéticos da sinterização de revestimentos cerâmicos de monoqueima do tipo BIIa", Cerâmica, v.57, n.344, pp.461-466, 2011.

[26] MEDEIROS, S.G., DUTRA, R.P.S., GRILO, J.P.F., et al., "Preparação de compósitos alumina-mulita de baixo custo via sinterização reativa entre uma argila caulinítica da Paraíba e hidróxido de alumínio", $C e$ râmica, v.62, n.363, pp.266-271, 2016.

[27] CELIK, H. "Technological characterization and industrial application of two Turkish clays for the ceramic industry”, Applid Clay Science, v.50, pp.245-254, 2010.

[28] TAHRI, W., SAMET, B., PACHECO-TORGAL, F., et al.,"Produtos de hidratação em argamassas geopoliméricas à base de argila da Tunísia para reparação de estruturas de concreto", Revista Matéria, v.21, n.1, pp.213-226, 2016.

[29] SANTOS, C. P., OLIVEIRA, H. A., OLIVEIRA, R. M. P. B., et al., "Caracterização de argilas calcárias utilizadas na produção de revestimentos cerâmicos no Estado de Sergipe - Brasil”, Cerâmica, v.62, pp.147$156,2016$.

[30] PAIVA, L. B., MORALES, A. R., DÍAZ, F. R. V., "Argilas organofílicas: características, metodologias de preparação, compostos de intercalação e técnicas de caracterização",Cerâmica, v.54, n.330, pp.213-226, 
2008.

[31] LUCIANO, R.V., ALBUQUERQUE, J.A., COSTA, A., et al., "Atributos físicos relacionados à compactação de solos sob vegetação nativa em região de altitude no sul do Brasil", Revista Brasileira Ciência do Solo, v.36, n.6, pp.1733-1744, 2012.

[32] MAHMOUDI, S., SRASRA, E., ZARGOUNI, F., “The use of Tunisian Barremian clay in the traditional ceramic industry: optimization of ceramic properties”, Applid Clay Science, v. 42, pp.125-129, 2008.

[33] CAMPOS, L. F. A., MACEDO, R. S., KIYOHARA, P. K., et al.,"Características de plasticidade de argilas para uso em cerâmica vermelha ou estrutural”, Cerâmica, v. 45, n.295, p. 295-302, 1999.

[34] VIEIRA, C. M. F., SOARES, T. M., MONTEIRO, S. N, "Massas cerâmicas para telhas: características e comportamento de queima",Cerâmica, v. 49, p. 245-250, 2003.

[35] MAROTO, J.M.M., AZCÁRATE, J.A.A., "An accurate, quick and simple method to determine the plastic limit and consistency changes in all types of clay and soil: The thread bending test",Applid Clay Science,v.114, pp.497-508, 2015.

[36] MENEZES, R. R., FERREIRA, H. S, NEVES, G.A., et al.,"Caracterização de argilas plásticas do tipo "ballclay" do litoral paraibano",Cerâmica, v. 49, n. 311, pp.120-127, 2003.

\section{ORCID}

Sileide de Oliveira Ramos

https://orcid.org/0000-0001-6353-1229

Gerbeson Carlos Batista Dantas

https://orcid.org/0000-0002-6662-5789

Helio de Lucena Lira

https://orcid.org/0000-0002-1527-9935

João Emanuell Araújo Marciano

https://orcid.org/0000-0003-3918-5412

Patrícia Mendonça Pimentel

https://orcid.org/0000-0002-3080-0688 